\title{
Surgical extraction of cardiac resynchronization therapy system with concomitant implantation of a new system with the use of epicardial leads in a patient with endocarditis
}

\author{
Jaroslaw Bis, MD, ${ }^{\text {a } K i n g a ~ G o s c i n s k a-B i s, ~ M D, ~}{ }^{\text {b }}$ Michal Krejca, MD, ${ }^{a}$ Elzbieta Zinka, MD, ${ }^{c}$ Janusz Skarysz, MD, ${ }^{a}$ \\ Leszek Machej, MD, and Andrzej Bochenek, MD, ${ }^{a}$ Katowice and Koszalin, Poland
}

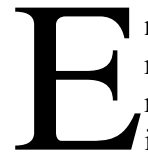

ndocarditis related to a transvenously implanted cardiac resynchronization therapy (CRT) system is a rare but serious therapeutic challenge, particularly in those patients in whom interruption of biventricular stimulation induces dramatic deterioration of their hemodynamic status.

\section{Clinical Summary}

We report the case of a 57-year-old man with a CRT system implanted transvenously in 2003 owing to dilative cardiomyopathy, in whom infective endocarditits with blood cultures positive for methicillin-resistant Staphylococcus epidermidis was diagnosed. The preoperative transthoracic echocardiogram revealed abnormal, moving echoes in the right atrium, impaired left ventricular ejection fraction of $20 \%$, moderate mitral regurgitation, and severe tricuspid regurgitation. Transesophageal echocardiography confirmed the presence of two large vegetations $(>1 \mathrm{~cm}$ in diameter) attached to the pacing leads within the right atrium (Figure 1). A temporary interruption of biventricular stimulation during preoperative echocardiography induced a deterioration of global systolic function, appearance of asynchrony, increase in mitral regurgitation, and after 15 minutes, signs of dyspnea and orthopnea. Consequently, the patient was qualified for a combined procedure of surgical removal of the infected CRT system with concomitant implantation of a new system with the use of epicardial leads.

The intervention was carried out with a median sternotomy approach, cardiopulmonary bypass, and cardioplegic arrest. After opening of the right atrium, the left ventricular lead proved to be firmly connected to the atrial wall by a tissue sleeve (Figure 2). Its mobilization was possible only after careful surgical separation. Subsequently, the distal ends of all three leads were completely removed from the heart and cut off at the level of the superior vena cava. The examination of the tricuspid valve did not reveal any pathologic condition necessitating surgical intervention. Bipolar epicardial leads (CapSure Epi 4968; Medtronic, Inc, Minneapolis, Minn) were implanted on the right atrium, right ventricle, and the lateral wall of the left ventricle within the most delayed segment according

From the First Department of Cardiac Surgery ${ }^{\mathrm{a}}$ and the Department of Electrocardiology, ${ }^{\text {b }}$ Medical University of Silesia, Katowice, Poland, and the Department of Cardiology, ${ }^{\mathrm{c}}$ District Hospital, Koszalin, Poland.

Received for publication Oct 7, 2007; accepted for publication Nov 21, 2007.

Address for reprints: Jaroslaw Bis, MD, First Department of Cardiac Surgery, ul. Ziolowa 47, 40-635 Katowice, Poland (E-mail: bisu@mp.pl).

J Thorac Cardiovasc Surg 2008;136:786-7

$0022-5223 / \$ 34.00$

Copyright (C) 2008 by The American Association for Thoracic Surgery doi:10.1016/j.jtcvs.2007.11.067

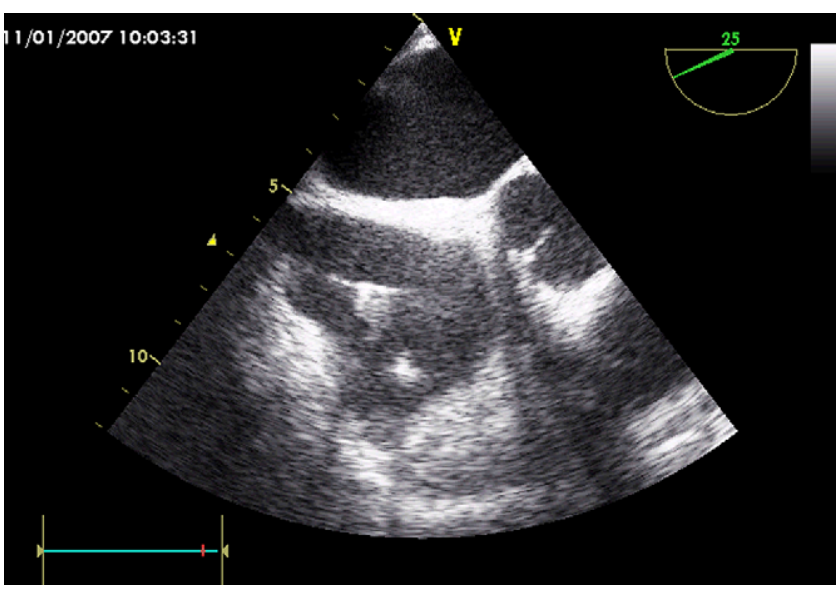

Figure 1. Transesophageal echocardiogram showing two vegetations greater than $\mathbf{1 0} \mathbf{~ m m}$ in diameter in the right atrium.

to the preoperative echocardiographic study. After release of the crossclamp, in the reperfusion period the pacing and sensing parameters were verified and subsequently the leads were connected to the biventricular pacemaker (Insync III; Medtronic) located in the abdominal wall. The biventricular stimulation was activated. After weaning from cardiopulmonary bypass, the sternotomy was closed routinely, and subsequently the old pacemaker was removed completely with the proximal ends of the old leads. The postoperative course was uneventful. The samples cultures confirmed the S. epidermidis etiology of the infection. After 30 days of antimicrobial treatment, the blood cultures were sterile and the patient's clinical status improved. In the predischarge echocardiogram, an improvement in CRT effect compared with preoperative status was confirmed with a left ventricular ejection fraction of $35 \%$ and a reduction in mitral and tricuspid regurgitation. The patient was discharged in very good general condition and leads a normal life.

\section{Discussion}

In recent years CRT has been proved to be an effective treatment option for patients with congestive heart failure and systolic dyssynchrony. ${ }^{1}$ However, the increasing implementation of CRT in clinical practice will result in a growing number of patients with endocarditis related to CRT pacing leads. It has been demonstrated that the only efficient treatment method of endocarditis on pacemaker leads is the removal of the entire pacing system. ${ }^{2,3}$ On the other hand, in some patients with advanced heart failure, the continuity of CRT therapy with its acute beneficial impact on systolic synchrony, diastolic filling time, and mitral regurgitation is crucial for maintaining hemodynamic stability. 


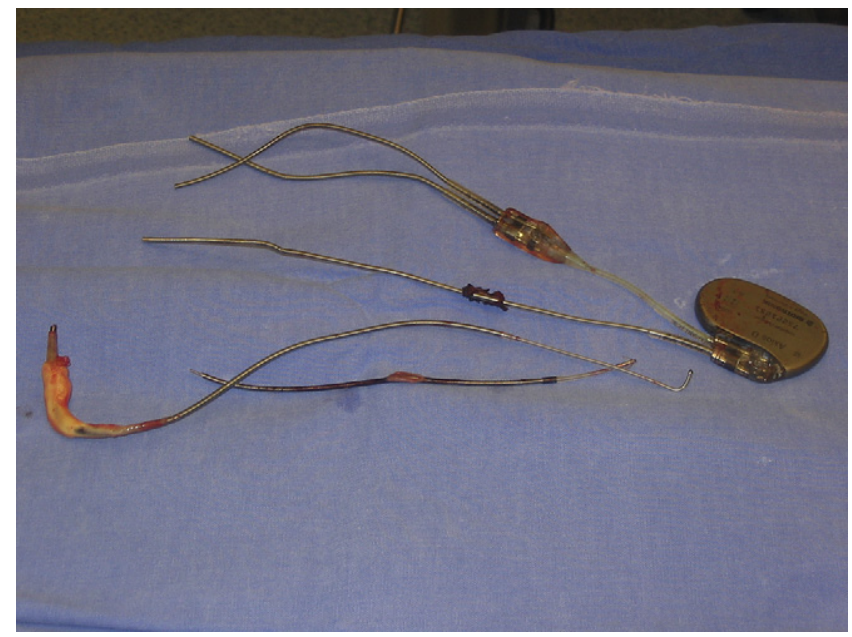

Figure 2. CRT system extracted from the patient.

In this setting, the surgical extraction of the CRT system with concomitant implantation of the new system with the use of epicardial leads may be a promising solution.

Moreover, the excellent exposition of the heart helps to overcome the limitations associated with coronary sinus anatomy, inasmuch as the left ventricular lead can be easily placed at the optimal site, which is of greatest importance for maximizing the benefit from CRT. ${ }^{4}$

We used bipolar nonpenetrating epicardial leads. The steroideluting tips prevent local fibrosis at the implantation site and ensure very good pacing and sensing parameters at long-term follow-up. ${ }^{4}$
Some authors advocate transvenous pacemaker lead removal regardless the size of vegetations. ${ }^{5}$ However, an extensive body of evidence supports the strategy of surgical extraction in case of vegetations exceeding $10 \mathrm{~mm}$ in diameter to prevent embolic complications. ${ }^{2}$ Moreover, in this case an attempt at transvenous removal could be risky considering the solid tissue sleeve connecting the left ventricular lead to the atrial wall (Figure 2).

In conclusion, we propose surgical extraction of the transvenously implanted CRT system with concomitant implantation of a new epicardial system as an effective treatment method of infective endocarditis, without the necessity of CRT interruption.

\section{References}

1. Vardas PE, Auricchio A, Blanc JJ, Daubert JC, Drexler H, Ector H, et al. Guidelines for cardiac pacing and cardiac resynchronization therapy: The Task Force for Cardiac Pacing and Cardiac Resynchronization Therapy of the European Society of Cardiology. Developed in Collaboration with the European Heart Rhythm Association. Eur Heart J. 2007;28:2256-95.

2. Klug D, Lacroix D, Savoye C, Goullard L, Grandmougin D, Hennequin JL, et al. Systemic infection related to endocarditis on pacemaker leads: clinical presentation and management. Circulation. 1997; 95:2098-107

3. Sohail MR, Uslan DZ, Khan AH, Friedman PA, Hayes DL, Wilson WR, et al. Management and outcome of permanent pacemaker and implantable cardioverter-defibrillator infections. J Am Coll Cardiol. 2007;49:1851-9.

4. Mair H, Sachweh J, Meuris B, Nollert G, Schmoeckel M, Schuetz A, et al. Surgical epicardial left ventricular lead versus coronary sinus lead placement in biventricular pacing. Eur J Cardiothorac Surg. 2005;27:235-42.

5. Ruttmann E, Hangler HB, Kilo J, Hofer D, Muller LC, Hintringer F, et al. Transvenous pacemaker lead removal is safe and effective even in large vegetations: an analysis of 53 cases of pacemaker lead endocarditis. Pacing Clin Electrophysiol. 2006;29:231-6.

\section{Interactive eLearning Activities \\ http://learning.ctsnet.org}

The Joint Council on Thoracic Surgery Education was pleased to introduce a series of unique eLearning activities to CTSNet users at the AATS annual meeting in Philadelphia. Sponsored by several cardiothoracic surgical groups, this exciting new educational tool contains narrated videos of actual surgical procedures followed by a series of questions and an evaluation to earn Continuing Medical Education credit.

* Diagnosis and Management of Complications of Mitral Valve Repair

* Off Pump Coronary Artery Bypass

* OPCAB Debriefing

* Pediatric Cardiopulmonary Bypass Emergency Situations

\section{Experience these new educational tools today and provide us with your feedback. Go to: http://learning.ctsnet.org}

William E. Baumgartner, Chair

Joint Council on Thoracic Surgery Education

Sponsored By:

American Association for Thoracic Surgery

The Society of Thoracic Surgeons

European Association for Cardio-Thoracic Surgery

Children's Memorial Hospital 